\title{
Design of Health Monitoring Program for Filling System based on Data Level Fusion
}

\author{
Cheng Long ${ }^{1}$, Xing Xiaochen ${ }^{2}$, Xie Weiqi ${ }^{1}$, Cheng Rui ${ }^{3}$ and Wang Lei ${ }^{3}$ \\ ${ }^{1}$ Department of Space Equipment, Equipment Academy, Beijing 101416, China \\ ${ }^{2}$ Department of Equipment Acquisition, Equipment Academy, Beijing 101416, China \\ ${ }^{3}$ Department of Graduate Management, Equipment Academy, Beijing 101416, China
}

\begin{abstract}
Aiming at filling system health monitoring, the health monitoring type partition based on the data level fusion is studied. The health monitoring type is mainly divided into two parts, fusion-threshold monitoring based on single sensor data and fusion monitoring based on multi-sensor data of the same type. On this basis, Single sensor fusion monitoring based on RTS-TA algorithm and Multi-sensor fusion monitoring based on improved weighted fusion algorithm are designed. For multi-sensor data fusion, the fusion schemes are designed respectively according to the different situation whether the initial accuracy is known or not.
\end{abstract}

\section{Introduction}

Test and launch ground system is mainly in charge of the spaceport ground facilities. To make a statistical of 70 typical failures during a period of test and launch ground system, which is shown as Fig. 1. During these failures, there are 48 ground facilities equipment failures (including software failures), $68.6 \%$ of all. And during the ground facilities equipment failures, the number of filling system failures is 25 , accounting for more than half of all the equipment failures.

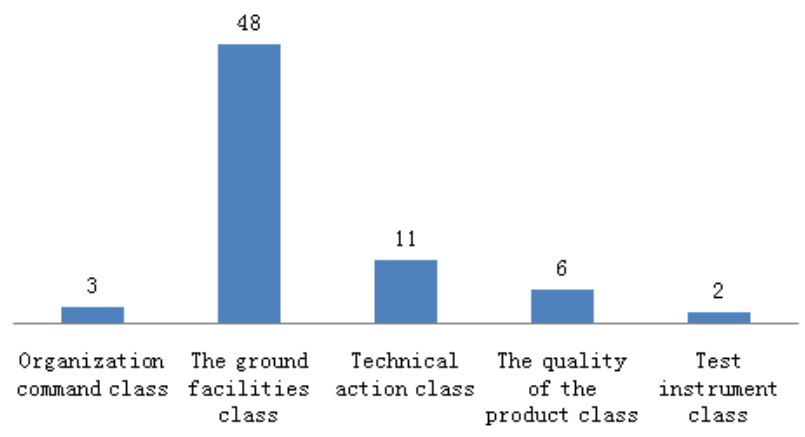

Figure 1. Statistical data of test and launch ground system typical failures

At the same time, as the propellant filling is often done before the launch within 24 hours. In this time, all the systems in the carrier rocket are in standby state. Whether the filling process can be completed accurately and safely within the given time or not, will directly affect the launch process. Once the filling failure appears, the loss is immeasurable. So the propellant filling is a key link in the process of launch site filling, and filling system is an important part of launch site ground equipments. Therefore, it is necessary to carry out the research on filling system health monitoring.

In order to complete the health monitoring of simple components such as tank, storage tank, valve and pipeline of filling system, the data information of pressure, temperature, liquid level and pipeline flow is collected. And the threshold discrimination is used for the health monitoring. The interior environment of filling system is very complex, so the single sensor test may be not useful because of the test or transmission errors. In this condition, it's difficult to guarantee the accuracy of the health monitoring for the appropriate component. To reduce the false alarm rate, the setting of threshold value is usually relatively broad compared with the normal components state. But in many cases, although the sensor component state information didn't exceed the threshold value, the component state has already been in a state of degradation in fact. In another word, person can conclude that the threshold value of monitoring method is not sensitive to the state of the component degradation.

In order to solve the problem of the low reliability of health monitoring for the threshold monitoring components of filling system, some sensors of the same type can be set up, which are used to monitor the component state at the same time. Adopting the data level fusion algorithm from information fusion technology to fusion the multi-sensors information, which can reduce the risk brought by he sensor test or transmission fault, and effectively improve the reliability and accuracy of component threshold monitoring.

In order to solve the difficult problem of threshold type component status deterioration monitoring of filling system, the forecasting technology is introduced into this 
paper, and single-sensor test information is used for the fusion in time dimension to finish the component predict health monitoring. Based on the state of a certain time period before the actual test information, the predict value of the time can be received. Using some rule to complete the predict value judgment, to make sure whether there is a clear prediction value mutation and whether the data can constitute an exception. For the anomaly data trends has been obtained, the state monitoring of component deterioration can be got based on some rules.

In summary, the health monitoring program for filling system based on data level fusion is designed in this paper.

\section{The health monitoring type partition based on the data level fusion}

The filling system health monitoring based on data level fusion technology can be divided into two types, one of them is fusion-threshold monitoring based on single sensor data and another one is fusion monitoring based on multi-sensor data of the same type.

Typically, the former has the high precision for sensor, and the threshold value is often set broader. In another conversation, when the parameters can be monitored for the same plurality of sensors, sensor accuracy requirement is general. At this time, in order to make sure that the monitoring information can truly reflect the actual health status of the component which is monitored, the multi-sensor data should be used.

\subsection{Fusion-threshold monitoring based on single sensor data}

Because of the physical constraints, some critical information of certain components in filling system can not meet (or not meet yet) the monitoring condition. In this conversation, the single sensor threshold monitoring type can be used only. The test data of a single sensor can be fused in time dimension, and the component health monitoring can be achieved by setting some judgment rule.

\subsection{Fusion monitoring based on multi-sensor data of the same type}

In order to collect the exact critical monitoring information of some components in filling system, such as the temperature, the pressure, the flow and the current information of the filling pump or the temperature, the level of propellant, multiple sensors will be set.

Usually, it can be divided into two parts. Aiming at the propellant level information, the health state can be monitored by threshold monitoring type. Aiming at the temperature and current information of the filling pump, the accuracy of the information collection can be improved based on multiple sensor data fusion.

The health monitoring type partition based on the data level fusion is shown as Fig. 2.

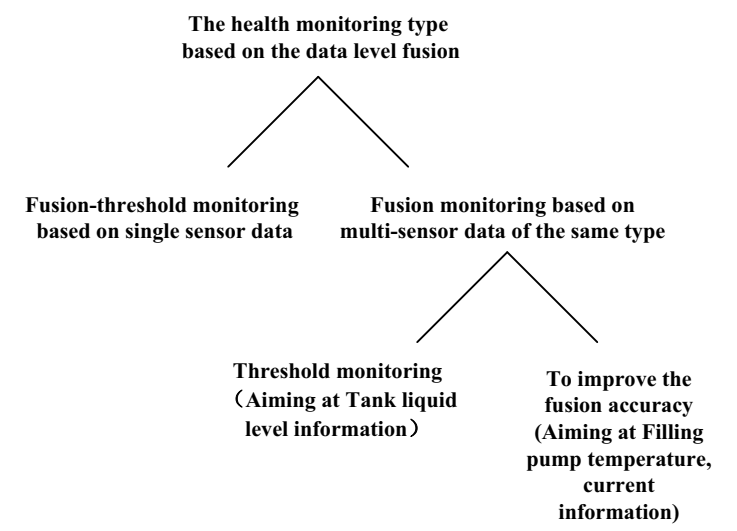

Figure 2. Statistical data of test and launch ground system typical failures

\section{Single sensor fusion monitoring based on RTS-TA algorithm}

At present, the health monitoring of some components in filling system still adopts manual testing and interpretation, therefore, it is necessary to add more operating positions and test personals. The increasing number of personnel and position adds the probability of the occurrence of malfunction caused by human factor, which brings harm to the implementation of the filling task. At the same time, the sensitivity of the artificial interpretation of health monitoring method for the sudden malfunction is poor, and the test efficiency is also relatively low. Therefore, by fusing the test data of the part state before the current moment and basing on prediction model, conducting the automatic prediction and interpretation for the status data in the follow-up unknown time is significant to the health monitoring for the threshold monitoring components of filling system. For the single sensor threshold monitoring, when the component state testing parameters are not yet exceeds a threshold, the prediction way to identify abnormal data, to analysis the trend of abnormal data, to complete the component state deterioration monitoring is adopted.

Time series analysis is a process based on the data information acquired of the present moment. It will analysis the inherent characteristics between data information, the relationship between data information and system disturbance, then establish a prediction model and predict the unknown data of the future unknown[1]. AR (p) model is one of the most widely used models in data prediction based on time series analysis. This model can be used to predict time series data when the data is stable, normal and zero mean. However, when using AR (p) model to predict the data, it is usually not predicted accurately and is pulled ahead for a few steps. Therefore, many scholars proposed using some algorithms and models to optimize the existing data before predicting and modelling for the data. Common optimization algorithms are such as neural network, support vector machine, genetic algorithm, particle swarm algorithm, ant colony algorithm and so on. The optimization algorithms above usually require large training sets, there are some shortcomings, such as local minimum, over learning and lack of theoretical guidance. It is difficult to adapt to real- 
time requirements and is not suitable for long-term data monitoring. The literature [2] proposed to apply Roll Time Series (RTS) algorithm for multi-step prediction of the condition monitoring data, using the latest predictive value continually to replace the data which first entered the prediction model, then conduct real-time correction to the prediction model parameters and improve the accuracy of the multi-step prediction.

In order to reduce the false alarm rate, the setting of threshold, combining with its own characteristics of the components to be monitored, is generally broader with respect to the normal component parameters for fusion threshold monitoring based on single sensor data. This mode of setting leads that, in some cases, although sensor test data has not yet exceeded the threshold, the trend of components' status monitoring information show that their health status has been degraded. It means that single sensor threshold monitoring isn't sensitive to the state deterioration or minor faults. When the component state parameter exceeds the threshold value, it usually indicates that an irreversible serious fault of the component is already happening.

When the component is normal, its condition monitoring parameter changes smoothly. By constructing some prediction model, a high-precision multi-step prediction of subsequent unknown parameters can be implemented. When the component state begins to degenerate, the state monitoring information will show a trend. This trend will break the autocorrelation of the condition monitoring information before and after, and get an inaccurate prediction result after the deteriorating state moment. Therefore, the method based on prediction can detect the deterioration of components state. And the occurrence of fault (or serious fault) can be avoided by take timely measures.

As the setting of threshold value is on the basis of comprehensive theoretical analysis and expert experience, its accuracy and reliability are high. In order to identify predicted outliers sensitively, the decision condition of in predictive algorithm is usually more demanding, and lead to that some normal state data is determined to be outliers. In fact, only the outliers with the presence of "close to trend" can be judged as a true exception. Based on the foregoing analysis, in order to meet the real-time, accuracy, high efficiency and other needs of priming system component health monitoring, this paper, combining the rapidity of AR (p) model with the accuracy of rolling algorithm and the analysis of the trend of data, proposes a kind of "rolling time series - trend analysis" (RTS-TA) algorithm. First, identifies the abnormal data of component state monitoring on the basis of the RTS algorithm. Then, analyses the trend of abnormal data. When abnormal data trend meet certain setting conditions, it means that an abnormal event has occurred, namely that component state appears deterioration.

\section{Multi-sensor fusion monitoring based on improved weighted fusion}

With the rapid development of information processing technology and computer technology, multi-sensor information fusion technology has received widespread attention in the remote sensing detection, intelligent transportation, fault diagnosis, pattern recognition and other fields and also has made many achievements[3-6]. Using multiple sensors to measure and integration the same parameter can significantly eliminate the negative consequences of sensor uncertainty and external disturbance noise and generate more accurate reliable test values than a single sensor [7-9]. Among them, the data fusion method based on weighted fusion, has high precision without a priori knowledge about the measurement data in the process of integration and fusion, and has a widely attention becaese of its optimal, unbiased and minimum mean square error (mse). How to make a good use of weighted fusion algorithm ,the key part is to determine the weights of each sensor data for the data fusion.

When the rocket filling system is performing filling task, The filling accuracy should be higher than $1 \%$. Especially in the filling taskof low temperature filling system, the low temperature propellant of liquid hydrogen or liquid oxygen susceptible to loss. When a large number of gas accumulated in the pipeline, it is very easy to cause a measure error of flow sensor, which can reduce the accuracy of sensor monitoring data and quantitative calculation, and bring hidden trouble for the execution of filling task. In addition, the document [10] pointed out that the failure of measurement device and sensor in filling system is a kind of failure mode which is not dllowed to ignore. Therefore, to conduct the same type multi-sensor data fusion technology research also has important practical significance. The present research condition of the same type multisensor data weighting fusion algorithm is summarized and analyzed, and an improved weighted fusion algorithm considering the sensor initial accuracy is proposed. The fixed distance evidence in evidence theory is introduced into the data fusion weight value calculation, which is used for the weighted fusion of multi-sensor.

According to three different conditions, the improved weighted fusion algorithm model can be divided into the following three parts, which is shown as Fig. 3:

1) The sensor initial accuracy is known;

2) The sensor initial accuracy is unknown, but can be calculated through the actual measurement data. Using the actual measurement data to calculate sensors accuracy also need to meet the following requirements:

(1) The state of the object under test in a long period of time domain basic will not change, and it meets (or approximate meets) the static measurement condition;

(2) The number of measured data which are used for calculating is enough, usually more than 20 ;

3) The sensor initial accuracy is unknown, and also cannot be calculated through the actual measurement data. In this case, regardless of the sensor precision, only to adaptive weighted fusion based on actual measurement data. 


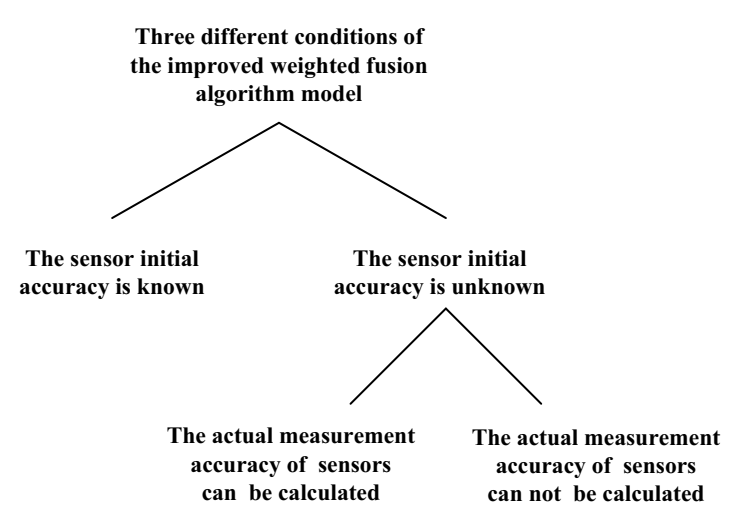

Figure 3. Three different conditions of the improved weighted fusion algorithm model

Suppose there are $n$ homogeneous sensors to measure an object (this paper considers only homogeneous sensor). The initial accuracy of these $n$ sensors are respectively $\sigma_{1}^{2}, \sigma_{2}^{2}, \cdots, \sigma_{n}^{2}$. The true value of the test object state is $X$, each sensor measurement data are respectively $x_{1}, x_{1}, \cdots, x_{n}$, the measurement data aevrage is $\bar{x}$, the estimate data after fusion is $\hat{x}$. The sensor can complete measurements alone, and the distribution of measurement data are independent of each other's condition.

According to the above three conditions, the improvement weighted fusion algorithm model is designed, which mainly includes the following steps, which is shown as Fig. 4:

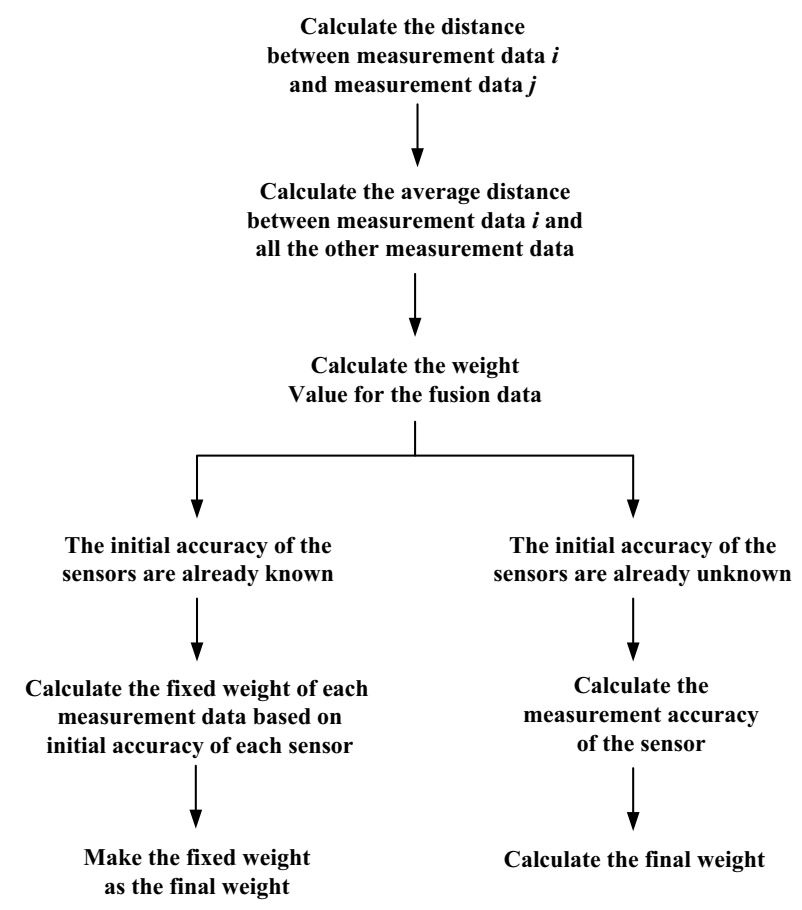

Figure 4. The improvement weighted fusion algorithm model

1) Calculating the distance between measurement data $\mathrm{i}$ and measurement data $\mathrm{j}$ based on fixed evidence distance, and person can get $d_{[c] i, j}$

$$
d_{[c] i, j}=\sqrt{\frac{x_{i}^{2}+x_{j}^{2}-2 x_{i} x_{j}}{x_{i}^{2}+x_{j}^{2}}} \quad i, j=1,2, \cdots, n
$$

2) Calculate the average distance between measurement data $\mathrm{i}$ and all the other measurement data based on $d_{[c] i, j}$, and person can get $d_{[c] i}$

$$
d_{[c] i}=\frac{1}{n-1} \sum_{j=1}^{n} d_{[c] i, j} \quad i, j=1,2, \cdots, n
$$

3) Calculate the weight value for the fusion data based on all the measurement data at the current moment, and person can get $w_{[c] i}$

$$
w_{[c] i}=\left(\frac{1}{d_{[c] i}}\right)^{3} / \sum_{i=1}^{n}\left(\frac{1}{d_{[c] i}}\right)^{3} \quad i, j=1,2, \cdots, n
$$

4) When the initial accuracy of the sensors are already known or the measurement accuracy can be calculated, person can get the final weight value $w_{i}$ based on measurement weight $w_{[c] i}$ and fixed weight. $w_{[g] i}$. If the initial accuracy of the sensor is unknown and also can't be calculated, make measurement weight to be the final weight value.

Among them, the fixed weight value calculation is divided into the following two cases:

(1) The initial accuracy of the sensors are already known

In this case, person can get the fixed weight of each measurement data based on initial accuracy of each sensor

$$
w_{[g] i}=\frac{1}{\sigma_{i}^{2}} / \sum_{i=1}^{n} \frac{1}{\sigma_{i}^{2}} \quad i=1,2, \cdots, n
$$

(2) The initial accuracy of the sensors are unknown but the calculation conditions are met

Colecting the historical measurement data about sensor $\mathrm{i}$, and in this paper person define the number as 20 . The historical measurement data are respectively $x_{i(j)}(i=1,2, \cdots, n ; j=1,2, \cdots, 20)$. The average data of the 20 historical measurement data is $\overline{x_{[s] i}}$, then the measurement accuracy of the sensor $\sigma_{[c] i}^{2}$ can be calculated, and then the fixed weight of each measurement data $w_{[g] i}$ can be calculated

$$
\begin{gathered}
\overline{x_{[l s] i}}=\frac{1}{20} \sum_{j=1}^{20} x_{i(j)} \quad i, j=1,2, \cdots, n \\
\sigma_{[c] i}^{2}=\frac{1}{20} \sum_{j=1}^{20}\left(x_{i(j)}-\overline{x_{[l]]}}\right)^{2} \quad i=1,2, \cdots, n \\
w_{[g] i}=\frac{1}{\sigma_{[c] i}^{2}} / \sum_{i=1}^{n} \frac{1}{\sigma_{[c] i}^{2}} \quad i=1,2, \cdots, n
\end{gathered}
$$

5) Then person can get the final fusion value $\hat{x}$ based on the weighted fusion of each measurement data using the final weight $w$

$$
\hat{x}=\sum_{i=1}^{n} w_{i} x_{i} \quad i=1,2, \cdots, n
$$




\section{Conclusion}

This paper has made a study of the health monitoring classification based on data level fusion, which are mainly divided into fusion-threshold monitoring based on single sensor data and fusion monitoring based on multisensor data of the same type. Aimming at single sensor data fusion-threshold monitoring, a kind of "rolling time series - trend analysis" (RTS-TA) algorithm based on A(R) model has been proposed. Aimming at fusion monitoring based on multi-sensor data of the same type, an improved weighted fusion algorithm considering the sensor initial accuracy is designed, which is divided into two parts: The sensor initial accuracy is known or unknown.

\section{References}

1. YANG Shu-ying, WANG Li-xian, NIU Ting-wei, et al. Multi-step prediction of rolling time series based on particle filter optimization. Journal of Systems Engineering and Electronic, 34(6):1097-1101, (2012)

2. LIU Hui, TIAN Hong-qi, LI Yan-fei. Short-term forecasting optimization algorithm for wind speed from wind farms based on wavelet analysis method and rolling time series method. Journal of Central South University (Science and Technology), 41(1):370-375, (2010).

3. Zhang Ting. Stress Identification on the Key Region of Large Span Space Structure, Harbin institute of technology, 2010.
4. Xiao Lizhi, Yang Dongping. The optimization algorithm of aviation equipment maintenance cost forecast and its applied research. Advanced Materials Research, 760:1851-1855, (2013)

5. Haibo Huo, Yi Ji, Shiming Wang, et al. The research on AdaBoost-BPNN model of point absorber wave energy converter. 2014 IEEE International Conference on Mechatronics and Automation, 8: 1762-1766, (2014).

6. Arkhom Songkroh, Rerkchai Fooprateepsiri, Woraphon Lilakiataskun. An intelligent risk detection from driving behavior based on BPNN and fuzzy logic combination. 13th International Conference on Computer and Information Science, 6, (2014).

7. Zhou Yao, Jiang Li-ping, LIU Yang. Data Fusion Algorithm Based on Support Degree under Interferential Environment. Fire Control \& Command Control, 39(3):12-14,19., (2014)

8. Mahler R. "Statistics 101" for multisensor, multitarget data fusion. IEEE A\&E SYSTEMS MAGAZINE, 19(1):53-64, (2004)

9. CHEN Shi-ming, CHEN Xiao-ling, XU Qing-gang, et al. Adaptive weighted fusion based linear distributed filtering algorithm. Control and Decision, 30 (4):605-610, (2015)

10. WU Xiao-tao, YANG Meng, YUAN Xiao-hui, et al. Bearing fault diagnosis using EEMD and improved morphological filtering method based on kurtosis criterion. Journal of Vibration and Shock, 34(2): 3844., (2015) 